\title{
Exposição de ciclistas ao ruído em uma cidade média brasileira
}

\author{
Cyclists' noise exposure in a Brazilian medium-sized city
}

Thiago da Cunha Ramos (https://orcid.org/0000-0002-0413-1724) ${ }^{1}$

Antônio Nélson Rodrigues da Silva (https://orcid.org/0000-0003-0996-8600) ${ }^{1}$

Léa Cristina Lucas de Souza (https://orcid.org/0000-0002-5770-4370) ${ }^{2}$

Luc Dekoninck (https://orcid.org/0000-0001-6910-4316) ${ }^{3}$

Dick Botteldooren (https://orcid.org/0000-0002-7756-7238) ${ }^{3}$

Inaian Pignatti Teixeira (https://orcid.org/0000-0002-9723-4297) ${ }^{1}$
${ }^{1}$ Departamento de Engenharia de Transportes, Universidade de São

Paulo. Av. Trabalhador Sãocarlense 400, Parque Arnold Schimidt. 13566590 São Carlos SP Brasil. thiagramos88@gmail.com ${ }^{2}$ Centro de Ciências Exatas e de Tecnologia, Departamento de Engenharia Civil, Universidade Federal de São Carlos. São Carlos SP Brasil.

${ }^{3}$ Grupo de Pesquisa Waves, Departamento de Tecnologia da Informação, Universidade de Gante. Gante Bélgica.

\begin{abstract}
This study aimed to assess cyclists' exposure to noise in a medium-sized Brazilian city. Mobile sensors were used to conduct noise measurements in streets with and without dedicated cycling infrastructures. The method can be summarized in the following procedures: i) characterization of the study area; ii) data collection and validation; iii) calculation of exposure indicators; and iv) comparison and representation of the results on maps. Two strategies were adopted for the analysis, namely, spatial data aggregation and temporal data aggregation. Thus, measurements were initially organized in 1,200 nodes distributed along the paths. The results indicate that bicycle riders in São Carlos may be exposed, in some routes, to a high proportion of high noise -level segments. In the two routes selected for this study, the cyclist was exposed to noise levels above the adopted threshold (> 75dBA) in 33.2\% and $18.9 \%$ of the nodes. Also, the possibility of simultaneously working with two related indicators has broadened the classification criteria of the route segments regarding noise exposure.
\end{abstract}

Key words Noise, Noise transportation, Noise measurement
Resumo O objetivo deste estudo foi avaliar a exposição dos ciclistas ao ruído em uma cidade média brasileira. Um sensor móvel foi utilizado para as medições de ruído em ruas com e sem infraestruturas cicloviárias segregadas. O método proposto contempla as seguintes etapas: i) caracterização da área de estudo; ii) coleta e validação de dados; iii) cálculo dos indicadores de exposição; $e$ iv) comparação e representação de resultados em mapas. Dois tipos de análises foram realizadas, por agregação espacial e por agregação temporal. Para isto, as medições foram inicialmente organizadas em 1.200 nós distribuídos nos percursos considerados. Os resultados apontam que os ciclistas que percorrem alguns trechos na cidade de São Carlos podem estar expostos a uma proporção considerável de ambientes com altos níveis de ruído. Nos dois trechos selecionados para este estudo, o ciclista esteve exposto à níveis acima do valor adotado (> 75dBA) em 33,2\% e 18,9\% dos nós. Além disso, observou-se que a possibilidade de trabalhar concomitantemente dois indicadores relacionados amplia os critérios de classificação dos trechos quanto à exposição do ruído.

Palavras-chave Ruído, Ruído dos transportes, Medição de ruído 


\section{Introdução}

O ruído e a cidade cresceram simultaneamente. Os arranjos urbanos, em constante mutação, apresentaram adensamento e espraiamento da malha viária nas últimas décadas ${ }^{1-3}$. Consequentemente, mais movimentos surgiram e a exigência por rapidez e eficiência nestes deslocamentos aumentou ${ }^{3}$. Na medida em que novas tecnologias de transportes se popularizavam, as cidades vieram a ser ocupadas massivamente por veículos motorizados $^{4-8}$. Aos poucos, o trânsito de pessoas e cargas se tornou ruidoso e nocivo'. Em consequência disto, ruídos superiores aos limites de tolerância para o ser humano se tornaram rotineiros $^{10}$.

Como alternativa ao tráfego intenso de veículos motorizados e ferramenta de promoção de bem-estar e saúde ${ }^{11-13}$, o modo cicloviário passou a ser incentivado nas cidades. No entanto, esse incentivo tende a ignorar o fato de que os usuários de bicicleta e os pedestres estão mais expostos aos gases e aos ruídos provocados pelo tráfego de veículos com motores de combustão interna ${ }^{14-16}$.

Contudo, observa-se que o ruído ganha importância no campo da saúde. A perspectiva isolada sobre a perda de audição foi substituída e ampliada para outros efeitos na saúde, principalmente aqueles associados à exposição prolongada ao ruído de tráfego. O ruído naturalmente provoca estresse ${ }^{17}$, que provavelmente interfere no sono ${ }^{18,19}$, afeta os sistemas nervoso e endócrino ${ }^{20}$, e há como resultantes: dificuldade de reprodução ${ }^{21}$, cognição ${ }^{22}$, além da pressão arterial ${ }^{23,24}$, ocorrência de infarto no miocárdio ${ }^{25,26}$, e até mesmo diabetes tipo dois $^{27}$ e mortalidade prematura $^{28}$.

A poluição sonora teve seus efeitos comparados aos que ocorrem na poluição atmosférica ${ }^{29,30}$. Diversos estudos têm pesquisado isolada ou concomitantemente o ruído juntamente com a poluição atmosférica, como aqueles conduzidos em onze cidades holandesas ${ }^{31}$, em Gante, na Bélgica $^{32}$, em Bangalore, na Índia ${ }^{33}$ ou em Montreal, no Canadá ${ }^{34}$. Dentre esses, observa-se o emprego de novas estratégias para medir o ruído, como as campanhas móveis realizadas em Gante ${ }^{35}$ e que podem ser associadas à aplicação de ferramentas de comunicação, tal como os telefones celulares, como foi feito em Cambridge, Inglaterra ${ }^{36}$.

No Brasil, o ruído é normalmente estudado em cidades de grande e médio porte, a partir de mapas sonoros de bairros residenciais, centrais e comerciais ${ }^{2,37-40}$. Outros estudos comparam a eficiência do tempo de medição do ruído em pontos fixos ${ }^{41}$, ou concentram-se na produção do ruído na interação entre o pneu e o pavimento ${ }^{42}$, no impacto à vizinhança dos ruídos gerados, sobretudo, em operações de terminais aeroviários, madeireiras, tráfego rodoviário, entre outros ${ }^{43-48}$. Por outro lado, estudos voltados para a paisagem sonora, compreendida como o ambiente acústico percebido e experimentado por uma pessoa em um determinado contexto ${ }^{49}$, demonstram que nem sempre o nível de ruído intenso representa incômodo ao receptor, pois depende da interrelação entre a pessoa, a atividade e o lugar, no espaço e tempo.

Considerando esse contexto, o objetivo desse trabalho é analisar a exposição de ciclistas ao ruído a partir da aplicação de campanhas móveis de medição, em distintos trechos cicloviários urbanos, com ou sem presença de infraestrutura cicloviária dedicada, em uma cidade brasileira de porte médio. Para isso, essa pesquisa estuda percursos que podem ser utilizados por ciclistas com destino ao trabalho e, consequentemente, considera o seu trajeto na ciclovia como parte de sua jornada de trabalho.

\section{Método}

A fim de analisar a exposição do ciclista ao ruído de tráfego urbano, esta pesquisa contempla as seguintes etapas: i) caracterização da área de estudo; ii) coleta e validação dos dados; iii) cálculo de indicadores de exposição e iv) comparação entre estes indicadores e representação dos resultados em mapas.

\section{Caracterização da Área de Estudo}

São Carlos, localizada no interior paulista, é uma cidade média com 244 mil habitantes es$\operatorname{timados}^{50}$. Nessa cidade, apesar do processo de implantação de ciclovias e ciclofaixas ocorrer desde 2012, a rede cicloviária proposta ainda se encontra incompleta. Por isso, este estudo considerou traçados de duas rotas (aqui denominadas Percursos), que englobam trechos da estrutura cicloviária existente e trechos da infraestrutura rodoviária que são utilizados pelos ciclistas como rotas (Figura 1).

O Percurso 1 é constituído por quatro infraestruturas cicloviárias, localizadas em áreas de interesse ambiental, devido à presença de cursos d'água. Além disto, contempla vias com potencial para realização de viagens por bicicletas, localizadas principalmente na área central da cidade, de 
particular interesse histórico. É composto por 12 trechos de vias (nomeados com letras e números de $1 \mathrm{~A}$ a $12 \mathrm{~A}$, sendo o número representativo da sequência em que foi percorrido o trecho e a letra representativa da posição do trecho, conforme Figura 1).

Quanto ao Percurso 2, é baseado essencialmente na demanda de viagens por bicicleta que ocorrem na mesma área central, mas em vias desprovidas de ciclovias ou ciclofaixas. O Percurso 2 é composto por 2 trechos de vias (nomeados trecho A e trecho B, conforme Figura 1).

Em resumo, os percursos estão compreendidos em uma zona de ocupação mista de uso do solo, fortalecida com as melhores condições de infraestrutura viária. Isto é, trata-se de uma área urbana consolidada, conforme exposto pelo Plano Diretor ${ }^{51}$.

Outros fatos marcantes para o presente estudo são: mais de metade da extensão do Percurso 1 se encontra em vias largas, com presença de áreas verdes junto a córregos. O Percurso 2 apresenta trechos cuja configuração urbana é constituída por ruas estreitas e fachadas relativamente altas.

\section{Coleta e Validação dos dados}

As coletas de dados foram realizadas sob a estratégia de campanhas móveis de medição, exclusivamente de ruídos. Para isso foi aplicado um sensor desenvolvido pelo grupo de pesquisa INTEC-Acoustics da Universidade de Gante, na Bélgica, calibrado para o registro de dados sob condições dos movimentos da bicicleta e com a capacidade de captação de dados no intervalo de 1 segundo, agregando à essas informações, as coordenadas geográficas do registro.

Para viabilizar as campanhas móveis de medição do ruído, adequou-se uma bicicleta para implantação do sensor móvel. Nesse caso, o sensor foi posicionado à frente do ciclista, para que o ruído preponderante fosse o de tráfego e não o do próprio usuário de bicicleta. Por essa razão, o suporte foi fixado sobre o guidão da bicicleta, forrado com materiais que reduzem o volume de vazios e suavizam os impactos da trepidação e da vibração (Figura 2).

Outro aspecto observado para as medições por bicicleta foi a restrição da velocidade máxima de deslocamento, que foi mantida no limite de $18 \mathrm{~km} / \mathrm{h}$, a fim de não acentuar o efeito do ruído aerodinâmico.

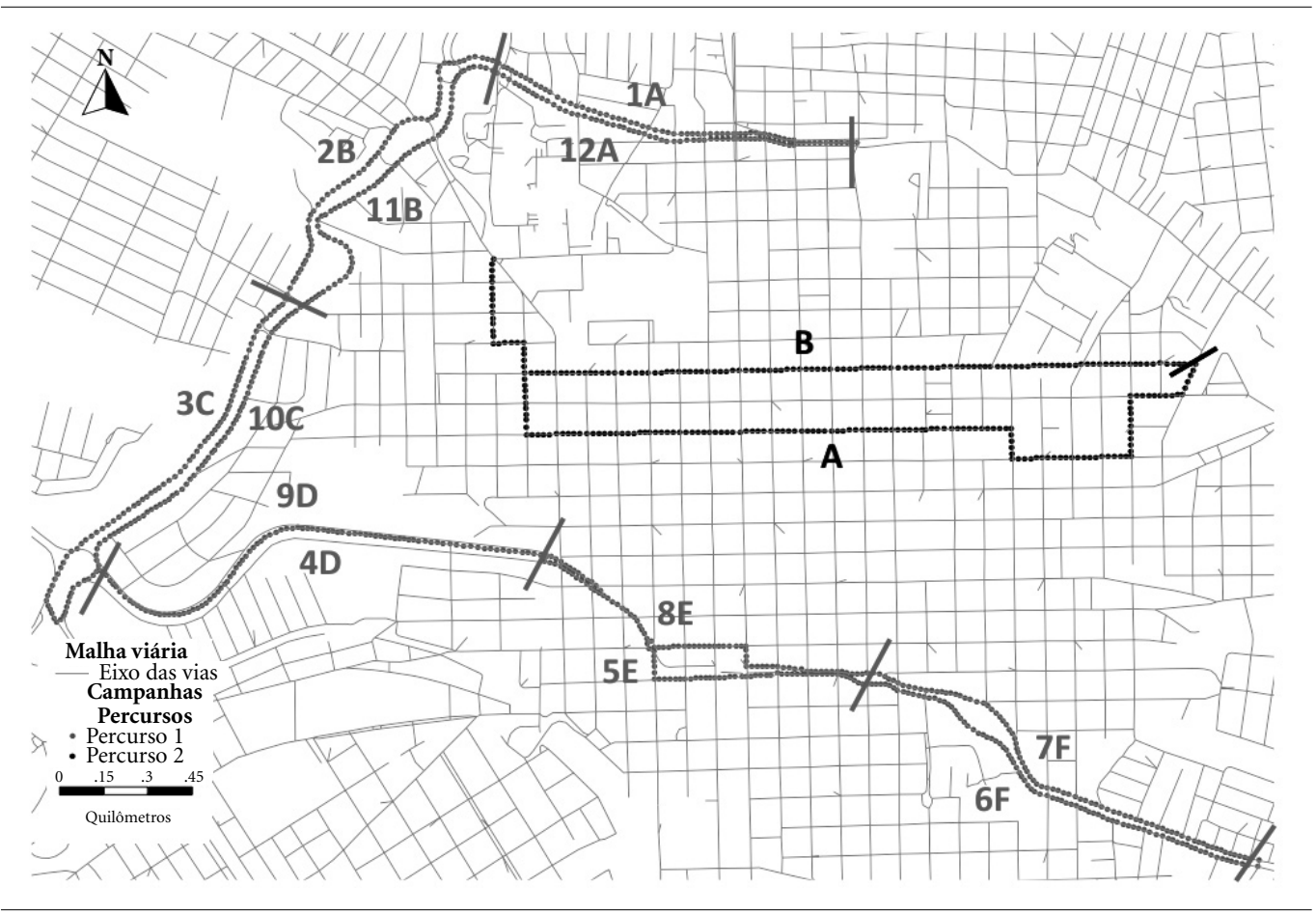

Figura 1. Rotas cicloviárias (com identificação de percursos e trechos) onde foram realizadas campanhas de medição de ruído em São Carlos-SP. 

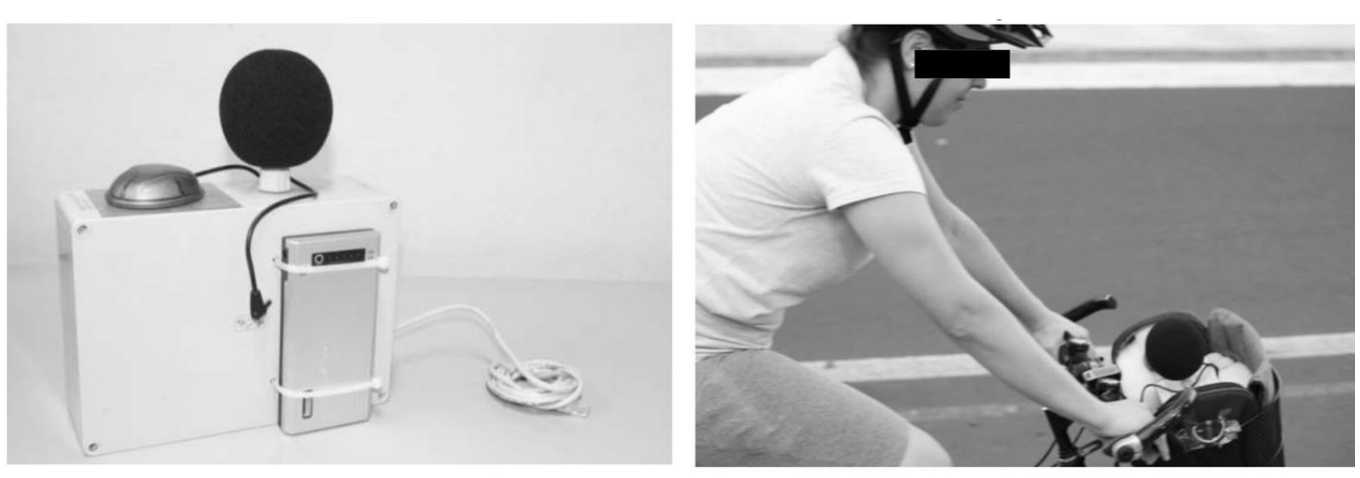

Figura 2. Sensor de ruído e seu posicionamento na bicicleta para as campanhas de medição em São Carlos-SP.

As viagens foram realizadas durante os horários de pico da manhã e tarde (7h30 às $8 \mathrm{~h} 30$ e $17 \mathrm{~h} 30$ às $18 \mathrm{~h} 30$, respectivamente), em dias típicos da semana (terça, quarta e quinta-feira). Após as viagens, foi realizada ainda uma validação de dados, levando em conta o intervalo entre registros de ruído, as coordenadas geográficas de cada ponto de medição, o registro cronológico e, fundamentalmente, a faixa de níveis de pressão sonora. Esse processo de validação procurou verificar a ocorrência de descalibração do sensor provocada pelo próprio deslocamento com a bicicleta. Outro aspecto observado nessa validação foi quanto aos eventuais problemas na coleta dos dados por GPS, referentes ao deslocamento da coordenada em relação à posição real.

Ao todo foram realizadas 15 campanhas móveis de medição do ruído, dentre as quais, oito viagens foram consideradas válidas. No total, foram computados 26.914 registros, divididos em: 22.221 referentes a 5 viagens no Percurso 1 e 4.693 referentes a 3 viagens no Percurso 2. O estudo foi aprovado pelo Comitê de Ética em Pesquisa envolvendo seres humanos da Escola de Artes, Ciências e Humanidades da Universidade de São Paulo.

\section{Cálculo de Indicadores}

Foram considerados dois indicadores para a análise dos níveis de pressão sonora: o Sound Exposure Level (SEL) e o nível equivalente sonoro contínuo $\left(L_{\text {Aeq }}\right)$, que podem ser descritos pelas equações 1 e 2 , respectivamente.
Equação 1:

$$
S E L=10 \log _{10}\left[10^{\left(\frac{l_{1}}{10}\right)}+\ldots+10^{\left(\frac{l_{n}}{10}\right)}\right]
$$

Onde: SEL - Sound Exposure Level (Nível de Exposição Sonora), em dBA; $l_{1}$ - primeiro nível sonoro, em dBA; $l_{n}$ - último nível sonoro, em dBA.

Equação 2:

$$
L_{\text {Aeq }}=S E L-10 \log _{10}\left(\frac{T}{T_{0}}\right)
$$

Onde: $L_{\text {Aeq }}$ - nível equivalente sonoro contínuo, em dBA; SEL - Sound Exposure Level (Nível de Exposição Sonora), em dBA; $T$ - tempo final de exposição; $T_{0}$ tempo inicial de exposição.

Na primeira etapa de análise, o indicador $L_{\text {Aeq }}$ foi calculado por agregação espacial, por pontos de referência (nós de agregação). Para isso, para cada nó de agregação foi considerada a média logarítmica dos diversos valores de $L_{\text {Aeq }}$ registrados dentro de um raio de $20 \mathrm{~m}$ ao redor do nó. Esse procedimento resultou em 1.200 nós, dos quais 926 foram distribuídos ao longo dos 12 trechos do Percurso 1 e 274 ao longo dos 2 trechos do Percurso 2. Esses nós foram numerados sequencialmente adotando-se números de 1 a 1.200.

$\mathrm{Na}$ segunda etapa de análise, a partir da coleta de dados contínuos, esses indicadores foram determinados pela agregação temporal, considerando os dados em três intervalos de tempo distintos: 5 segundos $\left(\mathrm{SEL}_{5 \mathrm{~s}}\right.$ e $\left.\mathrm{L}_{\text {Aeq, } 5 \mathrm{~s}}\right)$, 3 segundos $\left(\mathrm{SEL}_{3 \mathrm{~s}}\right.$ e $\left.\mathrm{L}_{\text {Aeq, } 3 \mathrm{~s}}\right)$ e 2 segundos $\left(\mathrm{SEL}_{2 \mathrm{~s}} \mathrm{e} \mathrm{L}_{\text {Aeq, } 2 \mathrm{~s}}\right)$. 


\section{Comparação e Representação dos Resultados em Mapas}

$\mathrm{Na}$ primeira etapa de análise, por agregação espacial, verificou-se, por trecho, a quantidade de nós que se encontram acima dos valores de 75 e 85 dBA (respectivamente, limites de ruído moderado e insalubre, conforme indicado na literatura $^{52}$ ). Determinou-se, assim, o número de ocorrências de nós acima desses limites, considerando-se o número de viagens realizadas para cada percurso.

Para a segunda etapa de análise de comparação por agregação temporal, foi verificada, para cada trecho, a quantidade de vezes que o valor dos indicadores ultrapassou $85 \mathrm{dBA}$ (limite de ruído insalubre). Posteriormente, os resultados dessas comparações foram plotados em um mapa indicativo das médias de porcentagens dos indicadores nos dois percursos estudados.

\section{Resultados}

Os resultados são aqui apresentados, considerando-se os dois tipos de análises realizadas, por agregação espacial e por agregação temporal.

\section{Análise por Agregação Espacial}

Os resultados da análise por agregação espacial (Tabela 1) revelaram que, no Percurso 1, o ciclista esteve exposto à níveis moderados de ruído em 33,2\% dos nós percorridos (> 75dBA), enquanto que no Percurso 2 essa exposição ocorreu em 18,9\% dos nós. Já a exposição a ruídos acima do limite de ruído insalubre ( $85 \mathrm{dBA}$ ) foi em 1,0 e 1,7\% dos nós nos Percursos 1 e 2, respectivamente. Dessa forma, nota-se que, em apenas $66,8 \%$ e $81,1 \%$ dos nós percorridos, o ciclista ficou exposto a níveis aceitáveis de ruído $\left(L_{\text {Aeq }}<\right.$ 75dBA).

Observando a Tabela 1, nota-se também que, em alguns trechos, houve uma repetição de nós moderados e insalubres ao longo de diferentes coletas, indicando pontos com problemas espe-

Tabela 1. Relação de ocorrências de nós com ruídos moderados (75 dBA) e nós acima do limite de ruído insalubre $(85 \mathrm{dBA})$ por trechos.

\begin{tabular}{|c|c|c|c|c|c|c|c|c|}
\hline \multicolumn{9}{|c|}{ LAeq - Nível Sonoro Equivalente Contínuo } \\
\hline \multirow{3}{*}{ Trecho } & \multicolumn{6}{|c|}{ Percurso 1} & & \\
\hline & Número & Dia 1 & Dia 2 & Dia 3 & Dia 4 & Dia 5 & \multicolumn{2}{|c|}{ Ocorrências } \\
\hline & do nó & tarde & tarde & manhã & tarde & manhã & $>75 \mathrm{dBA}$ & $>85 \mathrm{dBA}$ \\
\hline $1 \mathrm{~A}$ & 52 & 79,96 & 85,16 & 85,04 & 87,15 & 92,33 & 5 & 4 \\
\hline \multirow[t]{4}{*}{$3 \mathrm{C}$} & 2 & 88,95 & 82,93 & 86,66 & 87,97 & 85,30 & 5 & 4 \\
\hline & 3 & 90,62 & 84,44 & 86,93 & 88,87 & 87,97 & 5 & 4 \\
\hline & 78 & 85,26 & 85,63 & 83,13 & 87,99 & 85,71 & 5 & 4 \\
\hline & 79 & 86,49 & 85,23 & 82,52 & 88,19 & 85,13 & 5 & 4 \\
\hline \multirow[t]{3}{*}{$8 \mathrm{E}$} & 30 & 87,17 & 87,17 & 88,75 & 80,85 & 86,46 & 5 & 4 \\
\hline & 31 & 87,94 & 87,94 & 87,92 & 79,29 & 86,27 & 5 & 4 \\
\hline & 32 & 86,38 & 86,38 & 88,55 & 77,00 & 86,37 & 5 & 4 \\
\hline \multirow[t]{3}{*}{$10 \mathrm{C}$} & 3 & 79,60 & 88,44 & 86,51 & 86,92 & 85,86 & 5 & 4 \\
\hline & 36 & 87,43 & 83,11 & 85,15 & 95,31 & 87,33 & 5 & 4 \\
\hline & 58 & 81,03 & 92,23 & 85,01 & 85,44 & 87,12 & 5 & 4 \\
\hline \multicolumn{9}{|c|}{ Percurso 2} \\
\hline \multirow{2}{*}{ Trecho } & \multirow{2}{*}{$\begin{array}{c}\text { Número } \\
\text { do nó }\end{array}$} & \multirow{2}{*}{$\begin{array}{l}\text { Dia } 6 \\
\text { tarde }\end{array}$} & \multirow{2}{*}{\multicolumn{2}{|c|}{$\begin{array}{l}\text { Dia } 7 \\
\text { tarde }\end{array}$}} & & \multirow{2}{*}{$\begin{array}{l}\text { Dia } 8 \\
\text { tarde }\end{array}$} & \multicolumn{2}{|c|}{ Ocorrências } \\
\hline & & & & & & & $>75 \mathrm{dBA}$ & $>85 \mathrm{dBA}$ \\
\hline \multirow[t]{2}{*}{ A } & 102 & 86,88 & & 81,10 & & 88,14 & 3 & 2 \\
\hline & 143 & 90,68 & & 77,00 & & 91,05 & 3 & 2 \\
\hline \multirow[t]{4}{*}{ B } & 27 & 87,37 & & 84,88 & & 85,87 & 3 & 2 \\
\hline & 28 & 86,50 & & 85,66 & & 83,91 & 3 & 2 \\
\hline & 40 & 90,79 & & 85,67 & & 85,64 & 3 & 3 \\
\hline & 41 & 88,56 & & 85,49 & & 85,41 & 3 & 3 \\
\hline
\end{tabular}


cíficos e recorrentes. O trecho 1A, por exemplo, apresenta cruzamento para o qual uma das vias apresenta velocidade relativamente alta juntamente a um sentido em aclive; isto ocasiona frenagem e acelerações bruscas, ocasionando um acréscimo na exposição do ciclista a níveis maiores de ruído de tráfego. Os nós evidentes no trecho 3C se concentram primeiramente em um cruzamento semaforizado, que antecede um longo segmento cuja velocidade limite é de $60 \mathrm{~km} / \mathrm{h}$; logo, a aceleração é também brusca e ruidosa. Posteriormente, os outros dois nós se encontram em uma rotatória com diversos acessos que exigem os efeitos sucessivos de frenagem e de aceleração. $\mathrm{O}$ trecho $8 \mathrm{E}$ tem seus nós em uma via em aclive expressivo de $12 \%$ aproximadamente, com presença de ponto de ônibus e alta concentração de veículos motorizados. No caso do trecho 10C, a principal fonte de ruído foram os veículos automotores em alta velocidade, causada pela grande distância entre um cruzamento e outro.

Entre os nós do Percurso 2, as ocorrências diminuem e apenas seis nós dentre os trechos A e $B$ apresentam ocorrências de ruídos moderados e insalubres. Apenas dois nós do trecho B apresentaram três ocorrências acima do ruído de insalubridade. O trecho B constitui uma importante via de veículos motorizados para a cidade de São Carlos. Tantos os nós com três quanto com duas ocorrências ocorreram em um segmento onde a via é relativamente estreita, as fachadas adjacentes se constituem de muro alto de uma fábrica, além da existência de ponto de ônibus. Os nós de ruído insalubre do trecho A ocorreram por outros fatores relativos aos processos de frenagem e aceleração recorrentes nos cruzamentos. Por fim, é possível destacar que as médias das porcentagens de $L_{\text {Aeq }}$ para os três intervalos de tempo mantiveram-se praticamente estáveis em ambos os trechos deste percurso.

\section{Análise por Agregação Temporal}

Além dos aspectos espaciais, buscou-se avaliar a exposição do ciclista em função do tempo que o mesmo ficou exposto. Um exemplo da relação entre os indicadores temporais $\left(S E L_{53}, S E L_{33}\right.$, $S E L_{2 s}$ - Linhas cinza-claro) e os indicadores espaciais $\left(L_{\text {Ae }, 55}, L_{\text {Aeq, } 35}\right.$ e $L_{\text {Aeq, } 25}$ - Linhas cinza-escuro) pode ser observado na Figura 3. Nota-se que, quanto maior o intervalo de tempo considerado (5 segundos, neste caso), maiores os valores para o SEL. Contudo, independentemente do tempo adotado, o nível de exposição acumulada (SELsegundos $)$ evidencia mais claramente que a perma- nência prolongada do ciclista ao ruído foi mais determinante no grau de insalubridade do trecho. Porém, essa exposição fica pouco evidenciada se considerada apenas pelo $L_{\text {Aeq,segundos }}$.

Para melhor visualização e comparação entre os trechos, a Figura 4 apresenta um mapa em que as médias dos indicadores $(\mu \%)$ é ressaltada. Nesse mapa, dentre os indicadores $S E L_{\text {segundos }}$, apenas o $S E L_{2 s}$ foi destacado, pois, além de ser mais conservador, observou-se que esse já seria capaz de distinguir claramente os trechos quanto à exposição do ruído. No entanto, para o $L_{\text {Aeq,segundos }}$, considerou-se necessária a apresentação das médias das porcentagens para os três intervalos de tempo estudados.

Assim, para o Percurso 1, ambos os indicadores evidenciaram os trechos mais e/ou menos ruidosos. Os trechos 4D e 9D foram os trechos em que o ciclista sofreu menor exposição ao ruído, enquanto os trechos $3 \mathrm{C}$ e $10 \mathrm{C}$ foram os mais ruidosos.

Para o Percurso 2, o SEL teve um comportamento inequívoco e destacou o trecho B como o que mais expôs o ciclista ao ruído, em todos os intervalos de tempo estipulados. Nesse caso, a configuração urbana (rua estreita, muro alto e ponto de ônibus) justifica o prolongamento dos efeitos do ruído e a consequente diferença de 10 pontos percentuais entre as médias de $S E L$ dos trechos A e B.

$\mathrm{O}$ trecho $3 \mathrm{C}$ foi o que apresentou a maior quantidade de nós acima da insalubridade, porém suas médias de $S E L$ e $L_{\text {Aeq,segundos }}$ apresentam valores menores do que as do trecho 10C. Este último é o de maior exposição ao ruído pelo ciclista, com médias das porcentagens para o $S E L_{2 s}$ alcançando $51 \%$, enquanto as médias de $L_{\text {Aeq,segundos }}$ chegaram aos $29 \%$.

Em contrapartida, no contexto do Percurso 1, pode ser observado que os trechos $4 \mathrm{D}$ e 9D, que compartilham os mesmos nós nos dois sentidos de deslocamento, são os segmentos menos agressivos. Em uma via ciclável totalmente exclusiva, com vegetação e intervenção paisagística no entorno, ambos os trechos apresentam as menores médias de $L_{\text {Aeq,segundos }}$ e de $S E L_{25}$. A ligeira distinção entre esses dois últimos trechos pode ser explicada pela concentração do fluxo de tráfego motorizado encontrada no momento em que o conjunto ciclista/sensor circulava.

\section{Discussão}

A poluição sonora advinda do trânsito não é somente um "incômodo", uma vez que pode afetar 

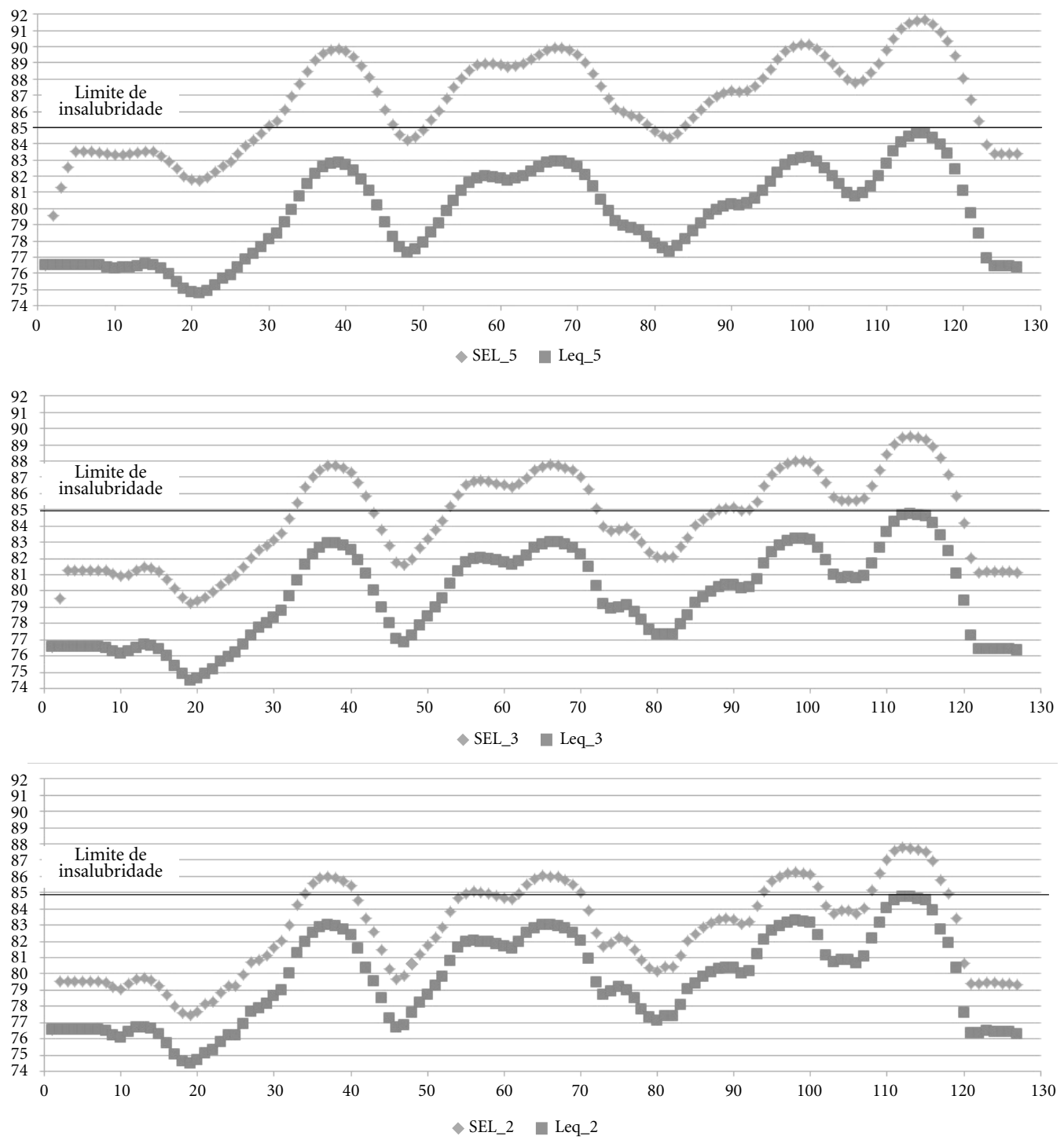

Figura 3. Comportamento de SEL e LAeq para cada intervalo de tempo.

SEL, LAeq x Tempo.

o bem-estar de forma geral. Os resultados dessa pesquisa apontam que os ciclistas que percorrem alguns trechos na cidade de São Carlos podem estar expostos a uma quantidade substancial de ambientes com altos ruídos. Verificou-se ainda que além do ruído pontual, caracterizado pelo $L_{\text {Aeq,segundos }}$ o tempo de exposição do ciclista a ambientes ruidosos também é importante para classificação da exposição do mesmo. Neste caso, quanto maior o tempo de exposição, mais insalubre. Vale destacar que os problemas associados a uma maior exposição ao ruído vão muito além dos problemas relacionados com o sistema auditivo ${ }^{53}$. Existem evidências relacionando a poluição sonora com hipertensão e problemas cardí$\operatorname{acos}^{54,55}$, desfechos de saúde mental, como baixa capacidade de concentração, comportamentos agressivos e índices elevados de stress ${ }^{56}$, entre outros.

Vale destacar que os efeitos nocivos do ruído à saúde não ocorrem apenas após uma exposição prolongada. Efeitos agudos como aumento da pressão arterial sistólica e diastólica, alteração na frequência cardíaca e liberação de hormônios 


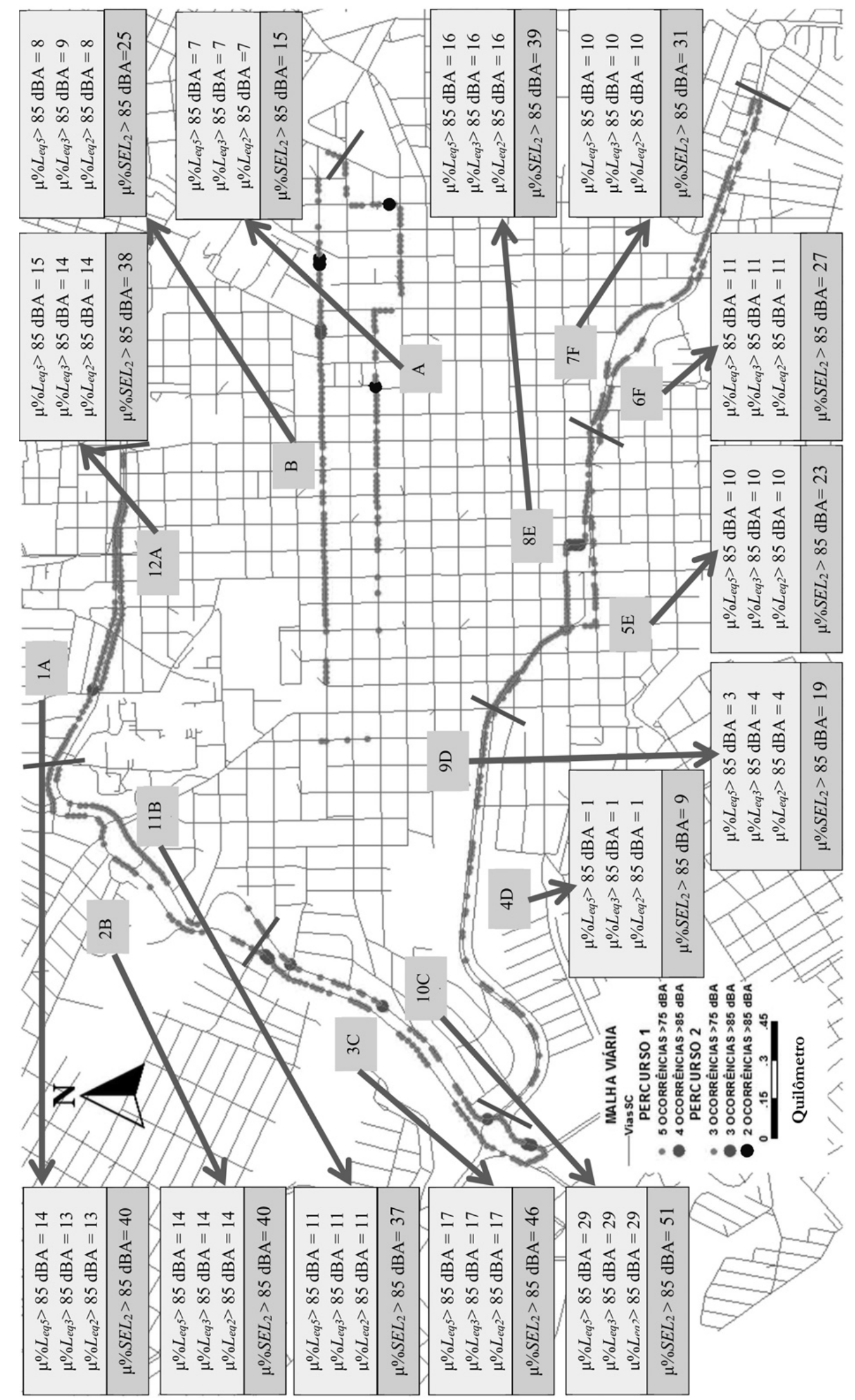

Figura 4. Nós de ruído insalubre e/ou moderado e apresentação das médias de porcentagens dos indicadores dos dois percursos analisados em São Carlos-SP, Brasil. 
relacionados ao estresse já podem ser notados a partir de um único evento ${ }^{57}$. Porém, os danos crônicos tendem a ser mais sistêmicos e complexos, como por exemplo o aumento nos fatores de risco para hipertensão arterial, dislipidemia, alteração na viscosidade do sangue e glicêmicas ${ }^{57,58}$.

Além disso, vale destacar que a fonte geradora desse ruído também influencia no desfecho de saúde pois, embora a exposição prolongada ao ruído seja prejudicial à saúde, as estimativas de risco para ruído ocupacional tendem a ser maiores do que aquelas para poluição sonora urbana ${ }^{59}$.

Nas cidades, grande parte da poluição sonora urbana está relacionada com a forma como as pessoas se locomovem pela cidade, principalmente pelas buzinas, frenagem e aceleração dos automóveis em geral. Logo, parece plausível que o incentivo para a substituição dos veículos automotores pelo transporte ativo poderia afetar de forma positiva na emissão desses ruídos. Porém, verifica-se que a utilização desses modos é acompanhada pela fragilidade de pedestres e ciclistas, que ficam sujeitos à poluição sonora e atmosférica.

Em um estudo realizado por Rabl e De Nazel$1 \mathrm{e}^{60}$, foi realizado uma estimativa do impacto da substituição do automóvel pelo transporte ativo (padrão de referência $=5 \mathrm{~km} /$ dia, 5 dias por semana). Assumindo custo relativo à saúde de 0,76 Euros por quilômetro, ao substituir o carro por bicicleta haveria um benefício de aproximadamente 1.800 Euros por ano, por indivíduo, gerado pela redução da poluição sonora.

\section{Pontos Fortes e Limitações do Estudo}

As campanhas móveis têm a vantagem de ser de baixo custo, requerendo um único conjunto ciclista/sensor para captar instantaneamente a ocorrência de níveis de pressão sonora durante a viagem. Em termos gerais, vale reforçar que a proposta de campanhas móveis de medição pode aprimorar a análise da exposição dos usuários de bicicleta, já que o equipamento de medição fica sujeito a condições semelhantes àqueles incidentes sobre o ciclista ao longo de seu trajeto. Além disso, a possibilidade de trabalhar concomitan- temente dois indicadores relacionados amplia os critérios de classificação dos trechos quanto à exposição do ruído. As porcentagens médias de $L_{\text {Aeq,segundos }}$ se mostraram como uma ferramenta clara ou simplificada de classificação. Entretanto, as relações de $S E L_{\text {segundos }}$ permitem distinguir sutilmente situações aparentemente semelhantes entre trechos.

Por outro lado, as campanhas móveis de medição têm, como aspecto negativo, a impossibilidade de se dissociar o ruído de tráfego rodoviário adjacente de outros ruídos decorrentes das demais fontes sonoras (como por exemplo, o ruído aerodinâmico, que ainda influencia na velocidade aplicada pelo ciclista, e os próprios ruídos gerados com os movimentos do conjunto ciclista/bicicleta).

\section{Conclusões}

A medição do ruído por campanhas móveis mostrou-se adequada e compatível com o contexto da exposição dinâmica do ciclista, de natureza variável, no tempo e no espaço. O armazenamento de dados a cada 1 segundo mostrou-se prático e viável para agrupamento de dados, em nós ou em intervalos de tempo, para análise do nível de exposição acumulada e do nível equivalente sonoro contínuo.

Embora praticamente todos os segmentos tenham apresentado níveis altos de ruídos, a aplicação do método em dois percursos distintos permitiu, até certo ponto, diferenciar a exposição de ciclistas em condições distintas do sistema viário urbano. Notou-se também que, a implantação de infraestrutura cicloviária em vias com amplos espaços livres, ao contrário do que poderia ser esperado, não significa necessariamente menor exposição do ciclista ao ruído. Como foi visto nos trechos mais ruidosos do Percurso 1, estes se encontram em uma avenida larga, com ciclovia em canteiro central a margear um córrego da cidade. Neste caso, a possibilidade de aplicar velocidade relativamente alta por parte dos veículos motorizados resultou em elevados níveis de ruído. 


\section{Colaboradores}

ANR Silva, TC Ramos, LCL Souza e D Botteldooren conceberam os experimentos. TC Ramos realizou a coleta de dados. L Dekoninck e D Botteldooren forneceram o sensor de ruído e realizaram o processamento dos dados com ele obtidos, bem como forneceram treinamento e suporte para a coleta e interpretação dos dados. TC Ramos, ANR Silva, LCL Souza e IP Teixeira conduziram as análises dos dados, interpretaram os resultados e redigiram o artigo.

\section{Agradecimentos}

Esta pesquisa foi apoiada pela Fundação de Amparo à Pesquisa do Estado de São Paulo (FAPESP), e Conselho Nacional de Desenvolvimento Científico e Tecnológico (CNPq). Este estudo também foi financiado em parte pela Coordenação de Aperfeiçoamento de Pessoal de Nível Superior-Brasil (CAPES).

\section{Referências}

1. Maia MAL. Contribuição ao mapeamento do ruído urbano na cidade de Porto Alegre - RS [dissertação]. Porto Alegre: UFRGS; 2003.

2. Ramos JOP. Dinâmica urbana na cidade de São Paulo o desafio do desenho das soluções acústicas [dissertação]. São Paulo: USP; 2007.

3. Balzan KL. Avaliação do ruído de tráfego veicular em área central de Chapecó - SC [dissertação]. Santa Maria: UFSM; 2011.

4. Ouis D. Annoyance from road traffic noise: a review. $J$ Environ Psy 2001; 21(1):101-120.

5. Piccolo A, Plutino, D Cannistraro, G. Evaluation and analysis of the environmental noise of Messina, Italy. Appl Acoust 2005; 66(4):447-465.

6. Singal SP. Noise pollution and control strategy. Oxford: Alpha Science International; 2005.

7. Rajakumara HN, Mahalinge Gowda RM. Road traffic noise prediction models: a review. Int J Sust Develop Plan 2008; 3(3):257-271.

8. Souza Filho JJ. Avaliação do ruído urbano na cidade de Campo Grande/MS [dissertação]. Campo Grande: UFMS; 2012.

9. Rodrigues F. Análise de ruído em terminais de transportes coletivos urbanos: desenvolvimento de modelos de previsão [dissertação]. Uberlândia: UFU; 2006.

10. Bortoli PS. Análise da poluição sonora urbana em zoneamentos distintos da cidade de Curitiba [dissertação]. Curitiba: CEFET-PR; 2002.

11. Hamer M, Chida Y. Active commuting and cardiovascular risk: a meta-analytic review. Prev Med 2008; 46(1):9-13.

12. Gordon-Larsen P, Boone-Heinonen J, Sidney S, Sternfeld B, Jacobs DR, Lewis CE. Active commuting and cardiovascular disease risk: the CARDIA study. Arc Int Med 2009; 169(13):1216-1223.

13. Hatzopoulou M, Weichenthal S, Dugum H, Pickett G, Miranda-Moreno L, Kulka R, Andersen R, Goldberg M. The impact of traffic volume, composition, and road geometry on personal air pollution exposures among cyclists in Montreal, Canada. J Exp Sci Environ Epidem 2013; 23(1):46-51.

14. Pucher J, Dijkstra L. Promoting safe walking and cycling to improve public health: lessons from the Netherlands and Germany. Am J Pub Heal 2003; 93(9):1509-1516.

15. Elvik R. The non-linearity of risk and the promotion of environmentally sustainable transport. Acc Anal Prev 2009; 41(4):849-855.

16. De Hartog JJ, Boogaard H, Nijland H, Hoek G. Do the health benefits of cycling outweigh the risks? Envir Heal Persp 2010; 118(8):1109-1116.

17. Michaud DS, Keith SE, Mcmurchy D. Annoyance and disturbance of daily activities from road traffic noise in Canada. J Acoust Soc Amer 2008; 123(2):784-792.

18. Omlin S, Bauer GF, Brink M. Effects of noise from non-traffic-related ambient sources on sleep: Review of the literature of 1990 - 2010. Noise Health 2011; 13(53):299-309.

19. Laszlo H, Mcrobie E, Stansfeld S, Hansell A. Annoyance and other reaction measures to changes in noise exposure - a review. Sci Total Environ 2012; 435-436: 551-562. 
20. Ising $\mathrm{H}$, Kruppa B. Health effects caused by noise: evidence in the literature from the past 25 years. Noise Health 2004; 6(22):5-13.

21. Ristovska G, Laszlo HE, Hansell AL. Reproductive outcomes associated with noise exposure - a systematic review of the literature. Int J Envir R Public Health 2014; 11(8):7931-7952.

22. Basner M, Babisch W, Davis A, Brink M, Clark C, Janssen S, Stanfeld S. Auditory and non-auditory effects of noise on health. Lancet 2014; 383(9925):1325-1332.

23. Babisch W, Wolf K, Petz M, Heinrich J, Cyris J, Peters A. Associations between traffic noise, particulate air pollution, hypertension, and isolated systolic hypertension in adults: the KORA study. Envir Heal Perspect 2014; 122(5):492.

24. Paunović K, Stanfeld S, Clark C, Belojević G. Epidemological studies on noise and blood pressure in children: observations and suggestions. Environ Int 2011; 37(5):1030-1041.

25. Peters A, Von Klot S, Heier M, Trentinaglia I, Hormann A, Wichmann HE, Lowel H. Exposure to traffic and the onset of myocardial infarction. $N$ Engl J Med 2004; 351(17):1721-1730.

26. Van Kempen E, Babisch W. The quantitative relationship between road traffic noise and hypertension: a meta-analysis. J Hypertens 2012; 30(6):1075-1086.

27. Dzhambov AM. Long term noise exposure and the risk for type 2 diabetes: a meta-analysis. Noise Health 2015; 17(74):23-33.

28. Halonen JI, Hansell AL, Gulliver J, Morley D, Blangiardo M, Fecht D, Toledano MB, Beevers SD, Anderson HR, Kelly FJ. Road traffic noise is associated with increased cardiovascular morbidity and mortality and all-cause mortality in London. Eur Heart $J$ 2015; 36(39):2653-2661.

29. Fritschi L, Brown L, Kim R, Schwela D, Kephalopolous S. Burden of disease from environmental noise - quantification of healthy life years lost in Europe. Geneva: WHO; 2011.

30. Hänninen $\mathrm{O}$, Knol AB, Jantunen M, Lim TA, Conrad A, Rappolder M, Carrer P, Fanetti AC, Kim R, Buekers, J. Environmental burden of disease in Europe: assessing nine risk factors in six countries. Environ Heal Perspect 2014; 122(5):439-446.

31. Boogaard H, Borgman F, Kamminga J, Hoek G. Exposure to ultrafine and fine particles and noise during cycling and driving in 11 Dutch cities. Atmospheric Environ 2009; 43(27):4234-4242.

32. Dekoninck L, Botteldooren D, Panis LI. An instantaneous spatiotemporal model to predict a bicyclist's black carbon exposure based on mobile noise measurements. Atmospheric Environ 2013; 79(2013):623631.

33. Dekoninck L, Botteldooren D, Panis LI, Hankey S, Jain G, Karthik S, Marshall J. Applicability of a noise-based model to estimate in-traffic exposure to black carbon and particle number concentrations in different cultures. Envir Int 2015; 74(2015):89-98.

34. Apparicio P, Carrier M, Gelb J, Séguin AM, Kingham S. Cyclists' exposure to air pollution and road traffic noise in central city neighbourhoods of Montreal. $J$ Trans Geog 2016; 57(2016):63-69.
35. Can A, Leclerq L, Lelong J. Dynamic estimation of urban traffic noise: influence of traffic and noise source representation. Appl Acoust 2008; 69(10):858-867.

36. Kanjo E. Noisespy: a real-time mobile phone platform for urban noise monitoring and mapping. Mob Net Appl 2010; 15(4):562-574.

37. Guedes ICM. Influência da forma urbana em ambiente sonoro: um estudo no bairro Jardins em Aracajú (SE) [dissertação]. Campinas: Unicamp; 2005.

38. Fritsch RC. Avaliação do ruído urbano: o caso da área central de Passo Fundo - RS [dissertação]. Passo Fundo: UPF; 2006.

39. Mardones MDM. Mapeamento dos níveis de ruído em Copacabana, Rio de Janeiro, através de simulação computacional [dissertação]. Rio de Janeiro: UFRJ; 2009.

40. Suriano MT, Souza LCL, Silva NAR. Ferramenta de apoio à decisão para o controle da poluição sonora urbana. Cien Saude Colet 2015; 20(7):2201-2210.

41. Mendonça ABD. Relações entre índices urbanísticos da forma urbana e a acústica ambiental [dissertação]. São Carlos: UFSCar; 2013.

42. Cabral MFF, Albuquerque FS, Freitas Neto O, Albuquerque TMA. Estudo dos mecanismos de geração de ruído de tráfego na interface pneu-pavimento. Transportes $2014 ; 22(1): 1-20$.

43. Nabinger LB. Medições de ruído aeronáutico dentro da área II do plano específico de zoneamento de ruído do aeroporto Salgado Filho, Porto Alegre/RS [dissertação]. Porto Alegre: UFRGS; 2005

44. Madruga JFS. Impacto sonoro das atividades madeireiras na qualidade de vida da população do bairro da Torre, João Pessoa, $P B$ [dissertação]. João Pessoa: UFPB; 2008.

45. Lessa BPV. Avaliação de poluição sonora em assentamento informal - estudo de caso comunidade Santo Marta - RJ [dissertação]. Rio de Janeiro: UFRJ; 2012.

46. Fiedler PEK. Poluição sonora nos eixos estruturais de transporte da cidade de Curitiba - PR [dissertação]. Curitiba: UFPR; 2013.

47. Jesus ADS. Impacto ambiental sonoro sobre a cidade de Águas Claras, Brasília-DF, com a construção da linha verde [dissertação]. Brasília: UnB; 2013.

48. Hecht VCES. Análise da incerteza na medição dos níveis de ruído aeronáuticos [tcc]. Rio de Janeiro: UFRJ; 2014.

49. International Organization for Standardization (ISO). ISO 12913-1:2014 Acoustics - Soundscape - Part 1: Definition and conceptual framework. Genebra: ISO; 2014.

50. Instituto Brasileiro de Geografia e Estatística (IBGE). IBGE - Cidades@ [Internet]. [acessado 2017 Jan 7]. Disponível em: http://www.ibge.gov.br/cidadesat/ topwindow.htm?1

51. Brasil. São Carlos. Plano Diretor. Lei n. ${ }^{\circ} 13.691$ de 25 de novembro de 2005. Diário Oficial da União; 2005.

52. Bistafa SR. Acústica aplicada ao controle de ruído. $2^{\mathrm{a}} \mathrm{ed}$ São Paulo: Blucher; 2011.

53. Berglund B, Lindvall T, Schwela D. Guidelines for Community Noise - World Health Organization [Internet]. [acessado 2017 Jan 7]. Disponível em: http:// www.who.int/docstore/peh/noise/Comnoise-3.pdf 
54. Babisch W, Beule B, Schust M, Kersten N, Ising H. Traffic noise and risk of myocardial infarction. Epidemio$\log y$ 2005; 16(1):33-40.

55. Van Kempen E, Kruize H, Bashuizen HC, Ameling $\mathrm{CB}$, Ataatsen BA, De Hollander AE. The association between noise exposure and blood pressure and ischemic heart disease: A meta-analysis. Environ Health Perspect 2002; 110(3):307-317.

56. Stansfeld S, Matheson M. Noise pollution: non-auditory effects on health. Br Med Bull 2003; 68(1):243257.

57. Babisch W. Cardiovascular effects of noise. In: Nriagu JO, editor. Encyclopedia of Environmental Health. Burlington: Elsevier; 2011.p. 532-542.

58. Babisch W. Cardiovascular effects of noise. Editorial. Noise Health 2011; 13(52):201-204.

59. Basner M, Babisch W, Davis D, Brink M, Clark C, Janssen S, Stansfeld S Auditory and non-auditory effects of noise on health. Lancet 2014; 383(9925):13251332.

60. Rabl A, De Nazelle A. Benefits of shift from car to active transport. Transport Policy 2012; 19(1):121-131.

Artigo apresentado em 26/10/2017

Aprovado em 27/11/2018

Versão final apresentada em 29/11/2018 